This is an author-created, un-copyedited version of an article published in European Journal of Physics. IOP Publishing Ltd is not responsible for any errors or omissions in this version of the manuscript or any version derived from it. The Version of Record is available online at https://doi.org/10.1088/0143-0807/36/3/035023.

\title{
A mechanical wave system to show waveforms similar to quantum mechanical wavefunctions in a potential
}

\author{
Sergej Faletič \\ University of Ljubljana, Faculty of mathematics and physics, Jadranska ulica 19, \\ 1000 Ljubljana \\ E-mail: sergej.faletic@fmf.uni-lj.si
}

\begin{abstract}
Interviews with students suggest that even though they understand the formalism and the formal nature of quantum theory, they still often desire a mental picture of what the equations describe and some tangible experience with the wavefunctions. Here we discuss a mechanical wave system capable of reproducing correctly a mechanical equivalent of a quantum system in a potential, and the resulting waveforms in principle of any form. We have successfully reproduced the finite potential well, the potential barrier and the parabolic potential. We believe that these mechanical waveforms can provide a valuable experience base for introductory students to start from. We aim at showing that mechanical systems that are described with the same mathematics as quantum mechanical, indeed behave in the same way. We believe that even if treated as a purely wave phenomenon, the system provides much insight into wave mechanics. This can be especially useful for physics teacher and others who often need to resort to concepts and experience rather than mathematics when explaining physical phenomena.
\end{abstract}

\section{Introduction}

While opinions between teachers differ regarding how appropriate using wave analogies in teaching quantum mechanics is, interviews with students suggest that many find analogies useful to create a mental picture and a mental picture necessary to grasp the underlying concepts [1]. We believe this especially applies to physics teachers and other physicists who often have to explain phenomena with concepts rather than mathematics and in a limited time. They have to drive from experience, and there is no experience available with wavefunctions. Additionally, the renown historical case of Maxwell tells us that great discoveries can be made with the help of an appropriate and consistent mental picture. In this article we study a mechanical wave system capable of correctly reproducing the waveform associated with a quantum mechanical system in a potential. We show that, in principle, it is possible to reproduce any shape of potential and the corresponding standing waveform. This is not an attempt to merely visualize the wavefunctions. These days there are numerous applets that do a much better job 
at that and are extensively interactive (see for example [2]). Instead we aim to show students that a mechanical system that is described mathematically in the same way as a quantum mechanical system, will indeed behave in the same way. We believe that this will strengthen students' trust in the formalism of quantum mechanics and hopefully make it easier for them to accept the consistent quantum mechanical picture faster. We further want to demonstrate that some feature of wavefunctions, which students encounter for the first time in the context of quantum mechanics, are actually inherent to all waves and not a quantum phenomenon at all. We primarily focus on the exponential tails and tunnelling waveshapes. In our experience, students never see these two features in classical waves and therefore may assume they are quantum phenomena. We believe that by observing the described system students will accept them as inherently wave features and remove them from what they consider quantum. This should allow them to focus on fewer new concepts and hopefully process them faster. Furthermore, it would be a wrongly built concept inventory, if they did end up considering them quantum phenomena. We discuss in depth a potential barrier with imaginary wavenumber and briefly show a finite potential well and the harmonic oscillator. In the end we discuss the didactical value of this approach. Even if the usefulness for teaching quantum mechanics is debated, these are still interesting and insightful wave phenomena in their own right. For example, we will see that it is necessary to provide an external force on the medium to correctly produce this kind of waveshapes.

\section{How to achieve a mechanical equivalent to a quantum mechanical system in a potential}

A quantum mechanical system is governed by the Schrödinger equation

$$
-\frac{\hbar^{2}}{2 m} \frac{\partial^{2}}{\partial x^{2}} \psi+V(x) \psi=\mathrm{i} \hbar \frac{\partial}{\partial t} \psi
$$

We assumed an arbitrary potential $V(x)$ as a function of the spatial coordinate $x$. Here, $t$ is time, $m$ is the mass of the particle, $\hbar$ is the reduced Plancks constant. To produce a corresponding mechanical system, we would need a wave equation of the form

$$
F \frac{\partial^{2}}{\partial x^{2}} y-\kappa(x) y=\mu \frac{\partial^{2}}{\partial t^{2}} y
$$

In this equation, $F, \kappa(x)$, and $\mu$ should be interpreted as general coefficients. In the general case we will use the term 'potential' for $\kappa(x)$, because it corresponds to $V(x)$ in the Schrödinger equation. It is clear that there is a difference in time evolution of both systems, but we will only consider the spatial part and the stationary states/standing waves. Therefore, this difference will not play a role in our discussion.

In our experiments we used a torsional wave-machine as the medium. For this case, the potential $\kappa(x)$ can be achieved by adding transversal elastic bands to the rods of the wave-machine, as seen on figures 1 and 2. Two bands are attached to each rod at distance $r$ from the central wire, and each to a fixed frame above and below. In the rest position they are already stretched keeping their net force on the rod zero. When 


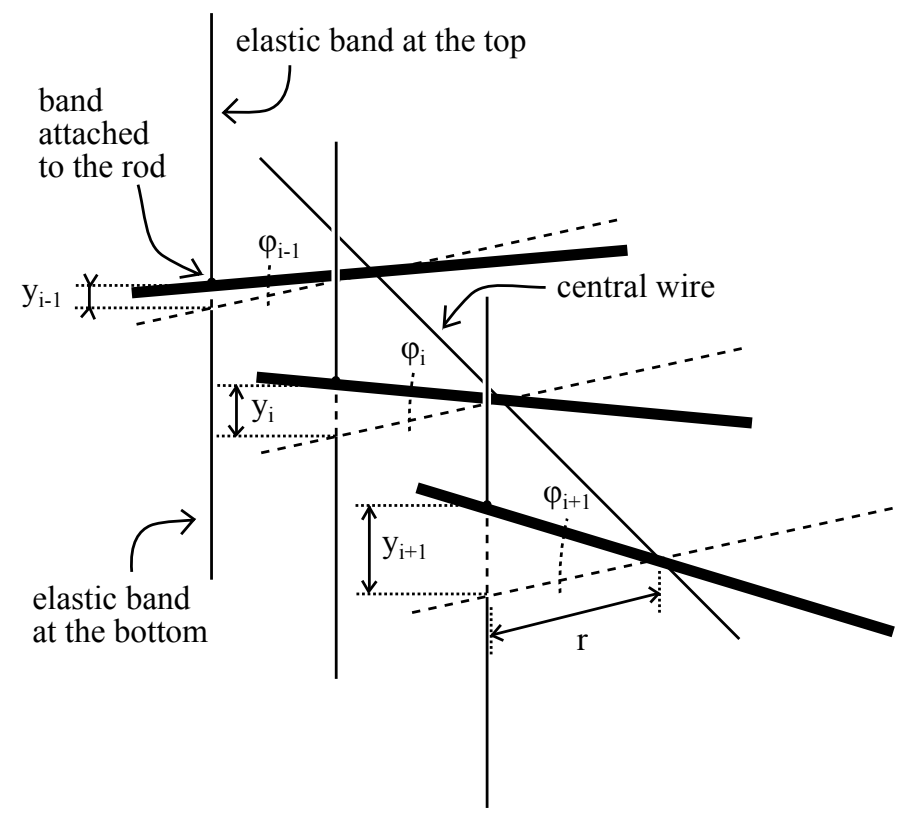

Figure 1. Sketch of part of the apparatus showing quantities used in the derivation of the wave equation.

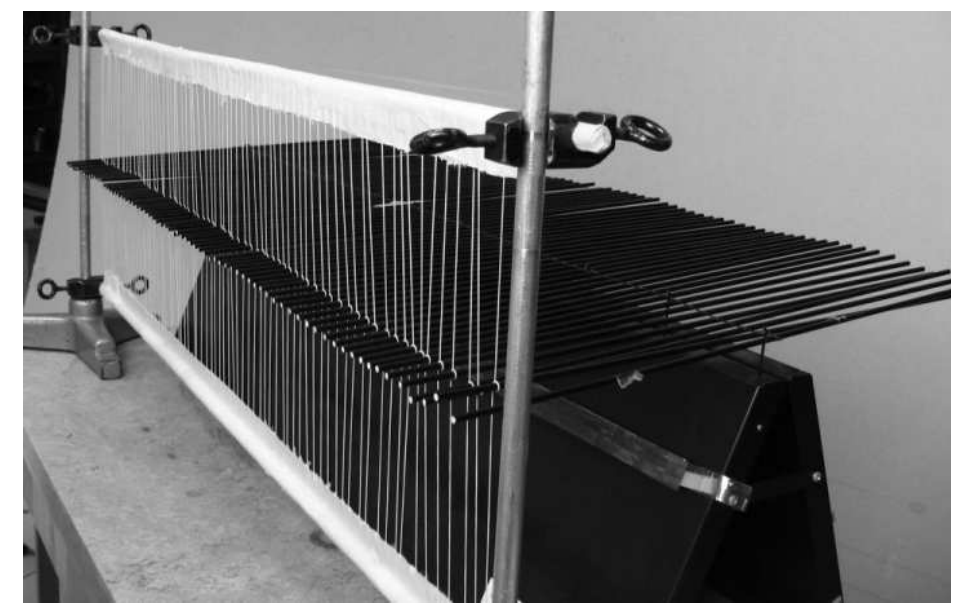

Figure 2. Picture of the apparatus with constant potential. By changing the spring coefficient of the elastic bands, the number of elastic bands per rod, or the position where they are bound to the rods we can achieve different forms of potential.

the rod is turned we assume they act as an ideal spring with spring coefficient $b$ and zero net force at rest. Results show that any nonlinearity does not visibly affect the waveforms. Let us rewrite (2) for such a case. With the torsional coefficient of the central wire denoted by $D$, and with $\Delta \varphi_{+1}=\varphi_{i+1}-\varphi_{i}$, and $\Delta \varphi_{-1}=\varphi_{i}-\varphi_{i-1}$, the combined torque of the neighboring rods on $\operatorname{rod} i$ is $M_{1}=\left(\Delta \varphi_{+1}-\Delta \varphi_{-1}\right) D$. Another torque is due to the elastic bands. With $y(r)=r \varphi_{i}$ being the vertical displacement of the attachment point (if $\varphi_{i}$ is sufficiently small), the force of the bands is $F_{s}=-b y(r)$, and the torque is $M_{2}=F_{s} r=-b r^{2} \varphi_{i}$. In general the bands on different rods can have different coefficients, and be attached at different distances $r$. We can then write 
$b=b(x)$ and $r=r(x)$. The wave equation is then

$$
\left(\Delta \varphi_{+1}-\Delta \varphi_{-1}\right) D-b(x) r(x)^{2} \varphi=I \frac{\mathrm{d}^{2} \varphi}{\mathrm{d} t^{2}} \quad,
$$

where $I$ is the moment of inertia of one rod. To recover the form similar to (2) we divide (3) by the distance between rods $\Delta x$. We rewrite $\Delta \varphi_{+1}-\Delta \varphi_{-1}=\Delta \Delta \varphi=$ $\Delta^{2} \varphi=\Delta x^{2} \frac{\Delta^{2} \varphi}{\Delta x^{2}}$. We introduce $\iota=\frac{I}{\Delta x}$, which can be thought of as the linear density of the moment of inertia, and

$$
\kappa(x)=\frac{b(x) r(x)^{2}}{\Delta x}
$$

We then get

$$
D \Delta x \frac{\Delta^{2} \varphi}{\Delta x^{2}}-\kappa(x) \varphi=\iota \frac{d^{2} \varphi}{d t^{2}} .
$$

To recover the continuous form, we let $\Delta x \rightarrow 0$. Then the number of rods approaches infinity, and the mass of one rod approaches zero. Thus, $I \rightarrow 0$, and $b \rightarrow 0$, keeping $\iota$ and $\kappa$ constant. We see that comparing this equation to the general form (2), $F=D \Delta x$, and $\mu=\iota$. In general the discrete version can be approximated by the continuous one if $\frac{\Delta x}{\lambda}<<1$. In our case, as seen from figures 3,4 and 5, we have $\frac{\Delta x}{\lambda} \approx 1 / 13$ at worst. We assume this is good enough, and use the continuous form where it is easier.

In case of a constant potential, $\kappa(x)=\kappa$, we arrive at the dispersion relation for propagating waves simply by inserting a solution of the form

$$
\varphi=A \cos (k x-\omega t)+B \sin (k x-\omega t)
$$

into (2). Introducing the travelling wave velocity without potential

$$
c_{0}^{2}=\frac{D \Delta x}{\iota}
$$

and the natural frequency of a lone oscillator comprised of one rod and the transversal elastic bands attached to it, without the coupling to other rods,

$$
\omega_{\kappa}^{2}=\frac{\kappa}{\iota}
$$

we get

$$
k(\omega)=\frac{\omega}{c_{0}}\left(1-\left(\frac{\omega_{\kappa}}{\omega}\right)^{2}\right)^{1 / 2} .
$$

With $c=\omega / k$ we can write

$$
\frac{1}{c^{2}}=\frac{1}{c_{0}^{2}}\left(1-\left(\frac{\omega_{\kappa}}{\omega}\right)^{2}\right)
$$

Gravel and Gauthier arrived at same result in [3], and Mouchet in [4], when discussing a classical use of the Klein-Gordon equation, which has the same mathematical form as (2) with $\kappa(x)=\kappa$. From (9) it can be seen that the wavenumber is imaginary whenever the natural frequency of a lone oscillator $\omega_{\kappa}$ is greater than the driving frequency. This corresponds in the quantum case to the potential being higher than the total energy of the particle. Considering the well known relation between energy and frequency in 
quantum mechanics, this is not surprising, but to make a quantitative comparison, one must take into account the differences between (1) and (2), which will not be discussed here.

By choosing different $b(x)$ and $r(x)$, we can produce potentials which are mathematically analogous to relevant systems in quantum mechanics.

\subsection{The standing waveforms}

Here we will limit ourselves to standing waveforms, which are analogous to quantum stationary states. Let us consider a potential formed of a series of potential steps. At each step we get a different wavenumber $k$. For springs that are too stiff $\left(\omega_{\kappa}>\omega\right)$, we get an imaginary wavenumber, as seen from (9). Inserting an imaginary $k$ into (6) produces an exponential form, rather then a sinusoidal one. The reflection produces the standing wave. The spatial part of the general solution in real notation to (2) on a segment with constant $\kappa(x)=\kappa$ is then

$$
\varphi(x, j)= \begin{cases}A_{j} \cos \left(k_{j} x\right)+B_{j} \sin \left(k_{j} x\right) & \text { for } \quad k_{j}{ }^{2} \geq 0 \\ A_{j} \exp \left(k_{j} x\right)+B_{j} \exp \left(-k_{j} x\right) & \text { for } \quad k_{j}{ }^{2}<0\end{cases}
$$

We can construct the waveform from segments of the form (11). The shape must be continuous and smooth. We must also meet the boundary conditions at the ends. We used numerical methods to find solutions, to keep the focus on the physics. Mathematics can be discussed separately. The actual methods were adapted to each particular case.

\section{The experiments, methods of analysis, and results}

We took a wave-machine of 72 rods. We used elastic bands found in common sewing elastic. Their spring coefficient could be modified by choosing the length of the band. The bands were attached to two support rods above and below the wave-machine with adhesive tape. In the middle they were wound up once around the rods, and also attached to them with adhesive tape. The apparatus set-up for a constant $\kappa$ can be seen on figure 2 .

\subsection{The potential barrier with imaginary $k$}

The potential barrier is a potential of the form

$$
\kappa(x)= \begin{cases}\frac{b r^{2}}{\Delta x} & \text { for } x_{1}<x<x_{2} \\ 0 & \text { elsewhere }\end{cases}
$$

We realised it by choosing eight subsequent rods in the middle of the wave-machine, and putting one vertical elastic string on each. We excited the wave-machine with different frequencies until we reached a stationary state with an imaginary $k$ inside the potential $\left(\omega_{\kappa} / \omega>1\right)$. We filmed the waveform with a high speed camera with 120 frames per second. We used video analysis software to determine the frequency and the shape of 
the waveform. We used the difference between the extreme positions as amplitude. This way we recovered a rest position that is horizontal and zero. Diamond-shaped points on figure 3 represent the standing wave amplitude for this situation. For the frequency we measured $\omega_{l}=(18.5 \pm 1.5) \mathrm{s}^{-1}$ on the left and $\omega_{r}=(18.4 \pm 1.4) \mathrm{s}^{-1}$ on the right, which match perfectly.

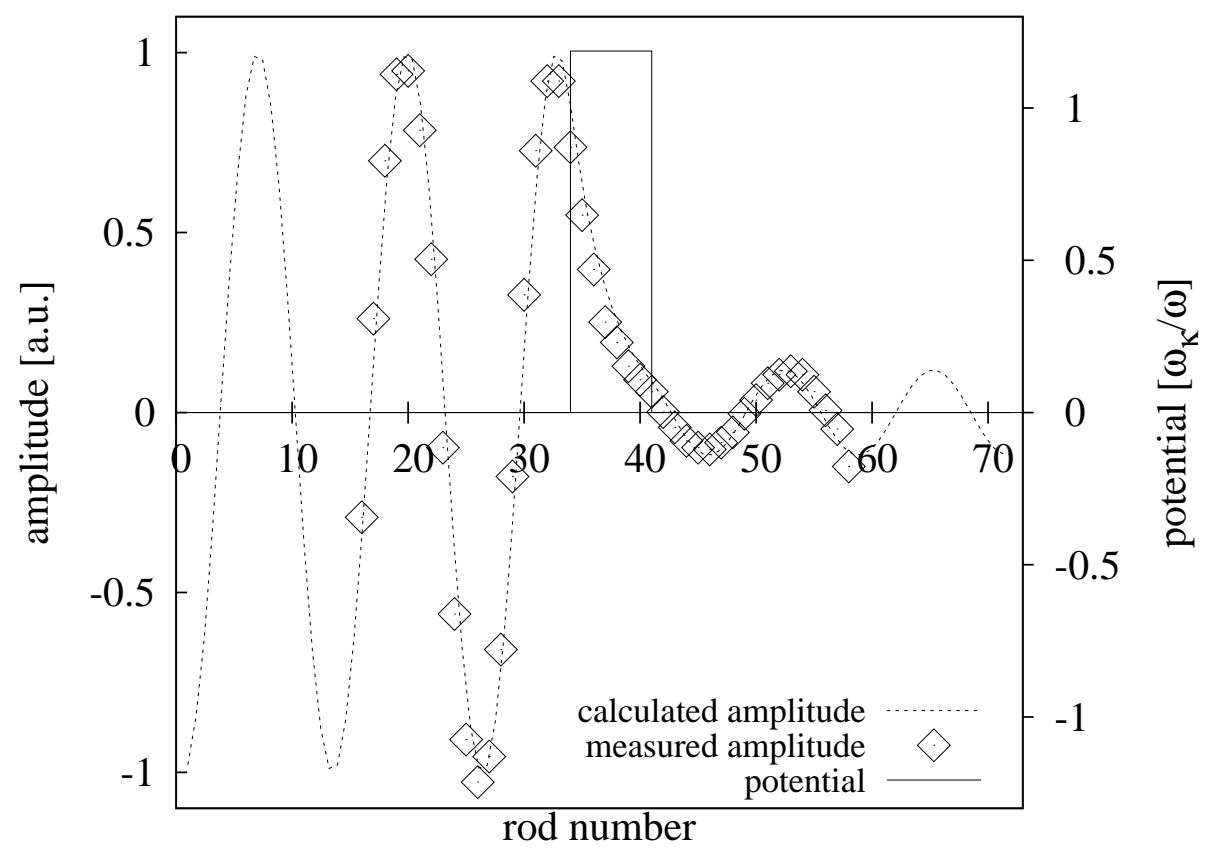

Figure 3. A wave travelling through a potential with an imaginary wavenumber. Measured values within the field of view of the camera (points) compared to the theoretical solution for the entire wave-machine (line).

We wanted to see how well theory matches with experiment. The spatial part of the theoretical solution for this case is of the form

$$
\varphi(x, j)= \begin{cases}A_{1} \cos \left(k_{1} x\right)+B_{1} \sin \left(k_{1} x\right) & \text { for } \quad x<x_{1} \\ A_{2} \exp \left(k_{2} x\right)+B_{2} \exp \left(-k_{2} x\right) & \text { for } \quad x_{1} \leq x<x_{2} \\ A_{3} \cos \left(k_{1} x\right)+B_{3} \sin \left(k_{1} x\right) & \text { for } \quad x \geq x_{2} .\end{cases}
$$

The value of $k$ on each segment depends on parameters $c_{0}, b, I$ and $\Delta x$, as seen from equations (7), (8), and (9). We measured the distance between rods $\Delta x=$ $(0.0127 \pm 0.0014) \mathrm{m}$ and determined $I=(0.783 \pm 0.005) \cdot 10^{-3} \mathrm{~kg} \mathrm{~m}^{2}$. We measured the wave velocity by measuring the frequency of the first three standing wave modes (without elastic bands) via high-speed camera and got $c_{0}=(0.474 \pm 0.010) \mathrm{m} / \mathrm{s}$.

We measured the frequency of a lone oscillator directly via high-speed camera. We connected the bands to one rod, disconnected from the central wire, and filmed its oscillation. We got $\omega_{\kappa}=(21.0 \pm 0.7) \mathrm{s}^{-1}$. The error was taken to be 10 frames per 287 frames in 4 oscillations filmed with 240 fps. We determined the spring coefficient $b$ independently by putting different weights on the elastic band, and measuring the natural frequency of the resulting oscillator. Values of $b$ differ for different frequencies 
due to nonlinearity of the material. For frequencies between $18 \mathrm{~s}^{-1}$ and $19 \mathrm{~s}^{-1}$, we got an average of $b=(3.91 \pm 0.07) \mathrm{N} / \mathrm{m}$.

We fitted the theoretical result (13) to the measured amplitude by adjusting the parameters $c_{0}, b$, and $\omega$. We set boundary conditions $\partial \varphi /\left.\partial x\right|_{x=0}=0$ and $\partial \varphi /\left.\partial x\right|_{x=x_{\max }}=0$ because we had both ends open.

One fit is shown in figure 3 with values $c_{0}=0.470 \mathrm{~m} / \mathrm{s}, b=3.9 \mathrm{~N} / \mathrm{m}$, and $\omega=18.0 \mathrm{~s}^{-1}$, which are all well within the range of the experimentally determined values $c_{0}=(0.474 \pm 0.010) \mathrm{m} / \mathrm{s}, b=(3.91 \pm 0.07) \mathrm{N} / \mathrm{m}$, and $\omega=(18.5 \pm 1.5) \mathrm{s}^{-1}$. The boundary conditions are also well met.

\subsection{Finite potential well}

For a finite potential well, $\kappa$ must be of the form

$$
\kappa(x)= \begin{cases}0 & \text { for }-a<x<a \\ \frac{b r^{2}}{\Delta x} & \text { elsewhere }\end{cases}
$$

The potential was realized as in the case of the potential barrier. The region with potential was the region where elastic strings were attached to the rods. The value of $k$ was imaginary. Figure 4 shows the measured standing waveforms (data points) compared to the theoretical waveform (lines), derived in the same way as described in section 3.1 .

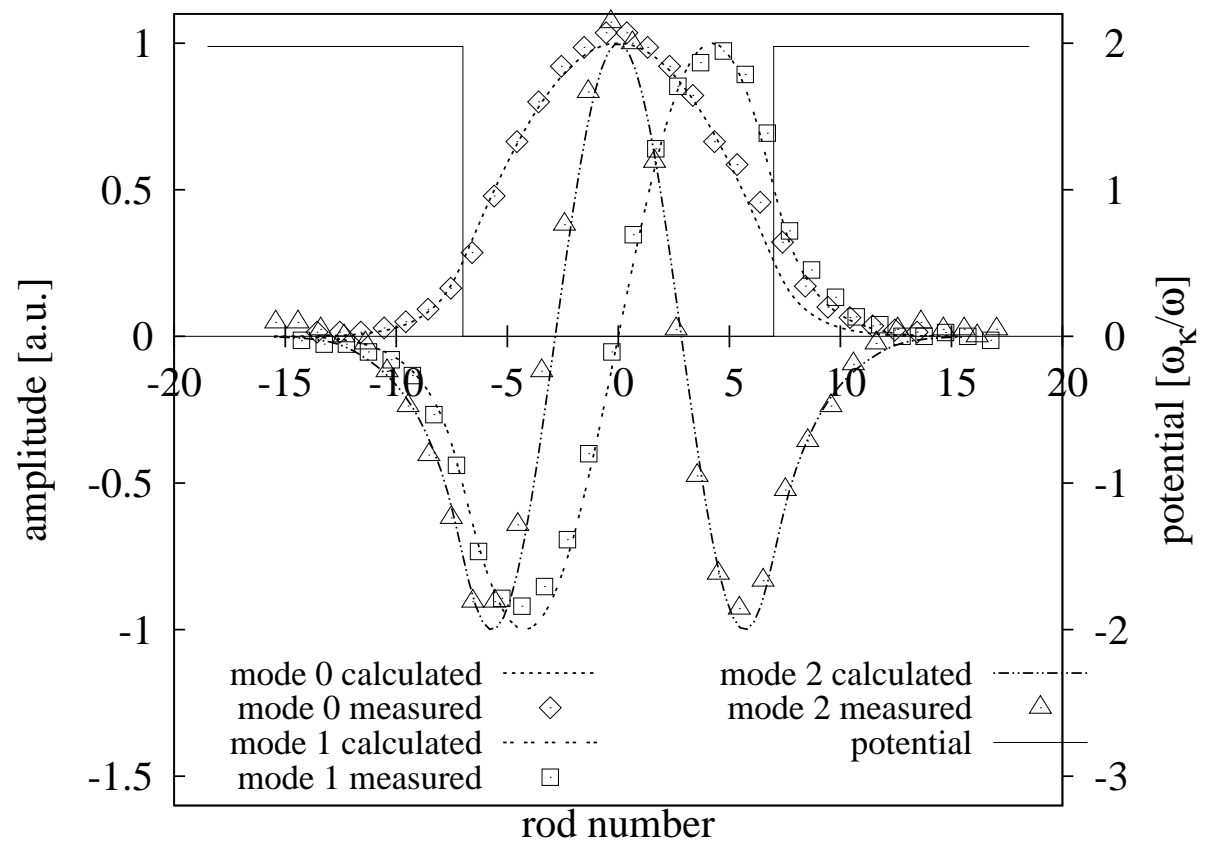

Figure 4. A comparison of the measured waveforms (points) to the theoretical shapes (lines) for a finite potential well. 


\subsection{Parabolic potential}

A parabolic potential is of the form $\kappa(x)=K x^{2}$, where $K$ is a constant. Using (4), we realized it with $b(x)=b$ and $r(x)=Q|x|$, where $Q$ is another constant. We only produced half of the shape, using the appropriate boundary conditions at $x=0$ to account for the symmetry of the shape. The theoretical waveform should be the same as the spatial part of the wavefunction of a quantum harmonic oscillator, which can be found in $[5,6,7]$.

$$
\varphi_{n}(x)=\left(2^{n} n !\right)^{-1 / 2} \pi^{-1 / 4} l^{-1 / 2} \exp \left(-\frac{1}{2} \frac{x^{2}}{l^{2}}\right) H_{n}\left(\frac{x}{l}\right),
$$

where $l$ is the characteristic length of the harmonic oscillator. We see that the solutions are real. $H_{n}$ is the $n$-th order (physicist's) Hermite polynomial, and $n$ is the index denoting different stationary states. We fitted the parameter $l$ and the amplitude to the data. We required $l$ to be the same for all modes, but we allowed a small fluctuation in the boundary conditions to account for our possibly imperfect driving. The magnitude allowed was such that the conditions were perfectly met in the interval $x=0 \pm \Delta x$. Figure 5 shows the results. Even though a full quantum harmonic oscillator-like system remains to be investigated, we hope this is enough to support our claim that it can be achieved.

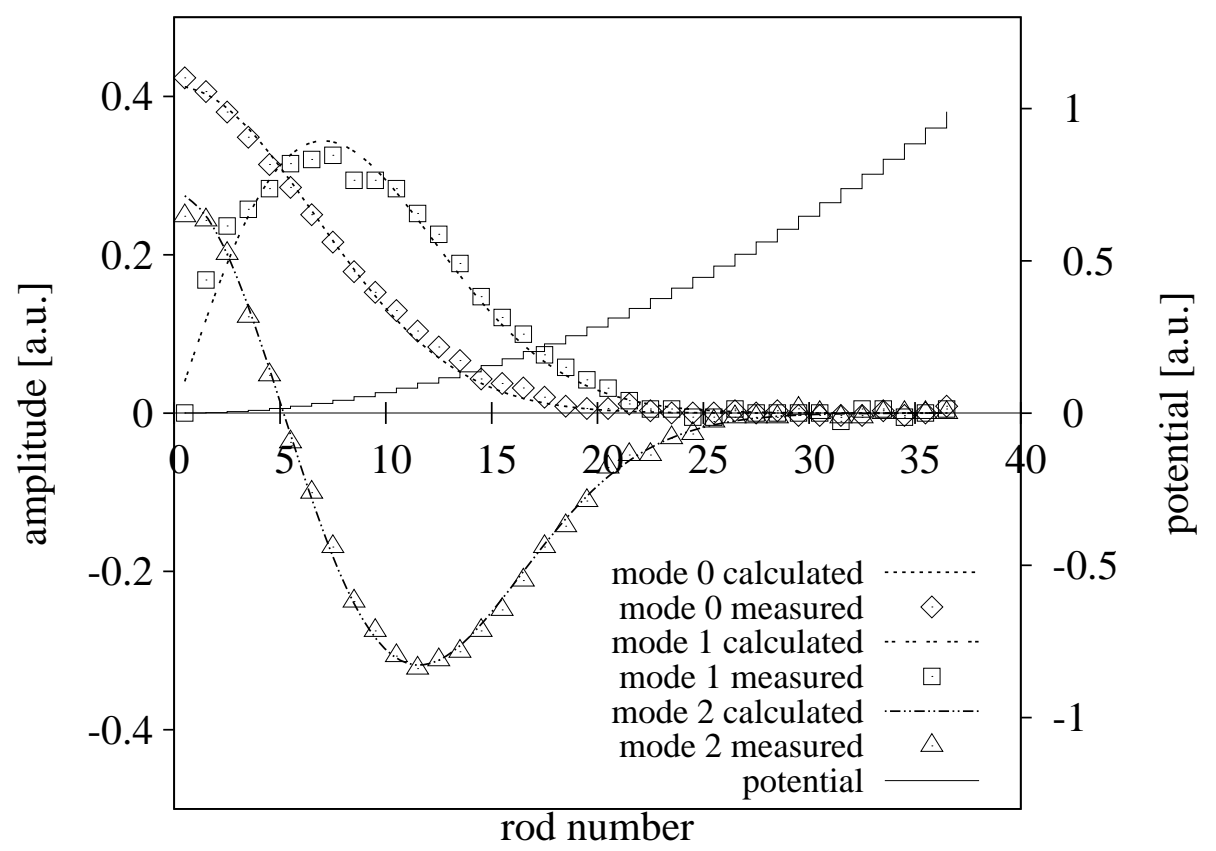

Figure 5. A comparison of the measured waveforms (points) to the theoretical shapes (lines) for half a parabolic potential. 


\section{Discussion of didactical value and conclusion}

In this paper we have shown that a mechanical equivalent of a quantum system in a potential can be achieved using external springs. We have shown three examples. We will now proceed to discuss how this system can be used to gain insight into the behaviour of waves and, by extension, wavefunctions. We want students to understand that these are inherently wave phenomena, with which they can have concrete experiences and they naturally carry over to wavefunctions. The concrete experiences should in a constructivist sense facilitate their building of formalism, which is necessary for the learning of quantum mechanics. On the other hand, the system provides tangible experience with the behaviour of waves which active and pre-service physics teachers can use when describing quantum phenomena to an audience with limited mathematical knowledge and who will not pursue quantum mechanics further.

We have shown that when the coefficient of the springs is too great $\left(\omega_{\kappa}=b / I>\omega\right)$ an imaginary $k$ is produced. The wave is not reflected at that position, but instead penetrates to some depth into such region as an exponential tail (seen in figures 3, 4, and 5). This is obviously inherent to wave mechanics. We believe that emphasis should be put into the interpretation of energy and potential in wave mechanics, and how it differs from particle mechanics. An infinitely steep step in potential is not like a wall as it is often interpreted. It is more like an area that strongly resists external influence. Similarly, the energy does not tell us how high something can jump, as kinetic energy of a particle would, but instead how much a wave can affect whatever it strikes. With this view it is much less counterintuitive, why a wave can penetrate a potential barrier to some depth, unless it is infinitely strong.

In further investigating the system, the wave-machine could be replaced with a slinky or a system of springs and beads. These systems lack flexibility in the parameter $r(x)$, but that could be replaced by an uneven length of the elastic bands.

\section{Acknowledgements}

I would like to acknowledge professor Martin Čopič for the discussion that prompted me to investigate this problem, professor Bojan Golli for valuable comments, professor Gorazd Planinšič for overall support, and Miha Kastelic for meticulously performing many of the experiments.

\section{References}

[1] Levrini O, Fantini P and Pecori B 2007 Proc. GIREP EPEC conf. 2007 (Rijeka) (Rijeka: Zlatni rez) p 319-24.

[2] PhET simulations (2015) https://phet.colorado.edu/en/simulations/category/physics/ quantum-phenomena (accessed 16 Feb 2015)

[3] Gravel P, Gauthier C 2011 Am. J. Phys. 79 p 447

[4] Mouchet A 2008 Eur. J. Phys. 29 p 1033

[5] Schwabl F 2007 Quantum mechanics 4th edn (Berlin: Springer-Verlach) p 47-53 
[6] Griffiths D J 1994 Introduction to Quantum Mechanics (New Jersey: Prentice Hall) p 31-6.

[7] Wikipedia, The Free Encyclopedia (2013) http://en.wikipedia.org/w/index.php?title= Quantum \_harmonic \_oscillator\&oldid=-558341649 (accessed 14 Jun 2013) 